\title{
Heterogeneity of non-cystic-fibrosis bronchiectasis in multiethnic Singapore: A prospective cohort study at a tertiary pulmonology centre
}

Si Ling Young ${ }^{1}{ }_{M R C P}$, Youxin Puan ${ }^{1}{ }_{M R C P}$, Si Yuan $\underline{\text { Chew }}{ }^{1}{ }_{M R C P}$, Haja Mohideen Salahudeen Mohamed ${ }^{2}{ }_{F R C R}$,

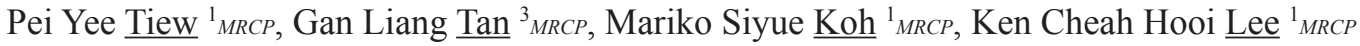

\begin{abstract}
Introduction: Non-cystic fibrosis bronchiectasis (NCFB) is a highly heterogenous disease. We describe the clinical characteristics of NCFB patients and evaluate the performance of Bronchiectasis Severity Index (BSI) in predicting mortality.

Methods: Patients attending the bronchiectasis clinic between August 2015 and April 2020 with radiologically proven bronchiectasis on computed tomography were recruited. Clinical characteristics, spirometry, radiology, microbiology and clinical course over a median period of 2.4 years is presented.

Results: A total of 168 patients were enrolled in this prospective cohort study. They were predominantly women (67.8\%), Chinese (87.5\%) and never-smokers (76.9\%). Median age of diagnosis was 64 years (interquartile range 56-71) and the most common aetiology was "idiopathic" bronchiectasis (44.6\%). Thirty-nine percent had normal spirometries. Compared to female patients, there were more smokers among the male patients $(53.8 \%$ versus $8.5 \%, P<0.001)$ and a significantly larger proportion with post-tuberculous bronchiectasis $(37.0 \%$ vs $15.8 \%, P=0.002)$. Fifty-five percent of our cohort had a history of haemoptysis. Lower body mass index, presence of chronic obstructive pulmonary disease, ever-smoker status, modified Reiff score, radiological severity and history of exacerbations were risk factors for mortality. Survival was significantly shorter in patients with severe bronchiectasis (BSI $\geq 9$ ) compared to those with mild or moderate disease $(\mathrm{BSI}<9)$. The hazard ratio for severe disease $(\mathrm{BSI} \geq 9)$ compared to mild disease (BSI 0-4) was 14.8 (confidence interval 1.929-114.235, $P=0.01$ ).

Conclusion: The NCFB cohort in Singapore has unique characteristics with sex differences. Over half the patients had a history of haemoptysis. The BSI score is a useful predictor of mortality in our population.
\end{abstract}

Ann Acad Med Singap 2021;50:556-65

Keywords: Bronchiectasis, exacerbations, gender, haemoptysis, mortality, Reiff score, sex

\section{INTRODUCTION}

Bronchiectasis is a chronic lung disease of significant morbidity and mortality. The pathological hallmarks of the disease are abnormal dilatation of airways resulting from recurrent inflammation, airway obstruction and mucous plugging. ${ }^{1}$ The past 2 decades have seen a significant increase in its prevalence, exceeding the threshold of 5 per 10,000 persons for the definition of an "orphan disease". ${ }^{2-7}$ In the UK, a rising incidence and prevalence was reported across nearly all age groups between 2004 and 2013, most notably among women above 70 years of age. ${ }^{2}$ A similar growing trend is reported in the US. ${ }^{3}$ There is less epidemiologic data on non-cystic fibrosis bronchiectasis (NCFB) in Asian countries. A cross-sectional survey from China reported a $1.2 \%$ prevalence of bronchiectasis among those aged 40 years and older. ${ }^{6}$ More recently, Choi et al. reported a prevalence of 464 patients per 100,000 person-years with NCFB in South Korea, with a mean age of $63.8 \pm 13.1$ years. ${ }^{7}$ These observations suggest that unlike cystic fibrosis that predominantly affects Caucasians, NCFB occurs commonly in both Caucasians and Asians, especially in the older age groups.

\footnotetext{
${ }^{1}$ Department of Respiratory and Critical Care Medicine, Singapore General Hospital, Singapore

${ }^{2}$ Department of Diagnostic Radiology, Singapore General Hospital, Singapore

${ }^{3}$ Department of Respiratory and Critical Care Medicine, Sengkang General Hospital, Singapore

Correspondence: Dr Si Ling Young, Department of Respiratory and Critical Care Medicine, Singapore General Hospital, Outram Road, Singapore 169608.

Email: siling.young@mohh.com.sg
} 


\section{CLINICAL IMPACT}

\section{What is New}

- This is one of the first studies describing the characteristics of non-cystic fibrosis bronchiectasis (NCFB) patients in Singapore, highlighting key features such as a high incidence of haemoptysis among these patients.

- The Bronchiectasis Severity Index (BSI) is a useful prognostic marker in our NCFB population.

\section{Clinical Implications}

- This study highlights the heterogeneity of NCFB and importance of further research to identify phenotypes that may help guide future management.

- The BSI can aid clinicians in their communication with NCFB patients regarding the prognosis of their disease.

Geographic variation in the aetiology and microbiology of NCFB has been described, such as the higher prevalence of idiopathic and post-infectious NCFB patients reported in European and Asian countries, ${ }^{8}$ compared to the US where NCFB was frequently associated with immune dysregulation. ${ }^{9}$ For microbiology, the rates of Pseudomonas aeruginosa and Hemophilus influenzae colonisation vary across the US, Europe and Asia Pacific region. Non-tuberculous mycobacterium (NTM) colonisation was found in $63 \%$ of NCFB patients in the US bronchiectasis research registry, ${ }^{10}$ but much lower rates were reported in Chinese studies. ${ }^{9}$ Other organisms like Klebsiella pneumoniae were significantly prevalent in NCFB patients in Thailand and South Korea. ${ }^{11,12}$

The heterogeneity of NCFB is further reflected in its diversity in clinical presentation, radiologic involvement, spirometry patterns and prognosis as reported by the various global registries on patients with NCFB. ${ }^{10,13-17}$ Such heterogeneity has led to a keen interest to identify phenotypes and endotypes with the aim of individualising treatment to improve outcomes. ${ }^{18}$ To date, information about the NCFB population in Singapore remains scarce. In this study, we describe the characteristics of NCFB patients in Singapore and evaluated the performance of Bronchiectasis Severity Index (BSI) in predicting mortality.

\section{METHODS}

Consecutive subjects (aged $\geq 21$ years) with diagnosis of bronchiectasis based on computed tomography
(CT), and attending the bronchiectasis clinic in Singapore General Hospital, a tertiary hospital in Singapore, were recruited into this prospective cohort study from 2017. The patients underwent a systematic evaluation of potential underlying aetiologies with a thorough assessment of disease symptoms, past history of sino-pulmonary infections including tuberculosis, ear infections, gastro-oesophageal reflux, subfertility, autoimmune disease and inflammatory bowel disease. Serum immunoglobulins and full blood count were performed for all patients, in accordance with the British Thoracic Society and European Respiratory Society guidelines. ${ }^{19,20}$ Other investigations such as autoimmune markers, alpha-1-antitrypsin level, and genetic testing for cystic fibrosis were performed if relevant clinical features were present. The aetiology of bronchiectasis was determined on the basis of the aforementioned investigations by the treating physician via a clinical-radiological approach. Spirometry results, respiratory microbiology and exacerbation history from time of diagnosis were also collected. Interpretation of spirometry was in accordance with the 2005 American Thoracic Society interpretative strategies for lung function tests. ${ }^{21}$ Bacterial colonisation was defined by the growth of the same bacteria on 2 or more occasions at least 3 months apart on either sputum or broncheo-alveolar lavage specimens.

The CT images were independently reviewed by an experienced thoracic radiologist, and the morphological characteristics and severity of bronchiectasis were determined. The modified Reiff score was used to assess the number of lobes involved and degree of dilatation. ${ }^{22,23}$ The left lingula was considered a separate lobe. The extent of bronchiectasis within each lobe was also graded with a score of 1,2 or 3 , according to the proportion of airways involved: $<25 \%, 25-50 \%$ and $>50 \%$, respectively. A radiology severity score was obtained by a summation of scores for all the lobes.

Exacerbation was defined as a deterioration in 3 or more of the following symptoms for at least 48 hours-cough, sputum volume or consistency, sputum purulence, breathlessness or exercise tolerance, fatigue and haemoptysis - associated with a requirement for treatment with antibiotics, which is modified from the original definition by Hill et al. ${ }^{24} \mathrm{~A}$ history of haemoptysis was defined as the patient having reported any amount of haemoptysis before in their lifetime that is attributed to bronchiectasis. Clinically significant haemoptysis referred to haemoptysis requiring bronchoscopy, intubation, bronchial artery embolisation or surgery. The BSI score was first derived and validated by Chalmers et al. and provided an easily 
Table 1. Clinical characteristics of patients with NCFB in Singapore and mortality subgroup analysis

\begin{tabular}{|c|c|c|c|c|}
\hline Characteristic & $\begin{array}{c}\text { Baseline characteristics } \\
\qquad(\mathrm{N}=168)\end{array}$ & $\begin{array}{l}\text { Mortality group } \\
\quad(\mathbf{n}=18)\end{array}$ & $\begin{array}{l}\text { Survival group } \\
\quad(\mathbf{n}=150)\end{array}$ & $P$ value \\
\hline Age at diagnosis, median (IQR), years & $64(56-71)$ & $56(54-60)$ & $64(57-71)$ & 0.693 \\
\hline BMI, median (IQR) & $19.3(17.3-21.8)$ & $18.5(18.1-21.5)$ & $19.3(17.3-21.7)$ & 0.003 \\
\hline Female, no. $(\%)$ & $114(67.8)$ & $11(61.1)$ & $103(68.7)$ & 0.517 \\
\hline \multicolumn{5}{|l|}{ Smoking status, no. (\%) } \\
\hline Never & $123(76.9)$ & $8(53.3)$ & $113(78.5)$ & 0.030 \\
\hline Active & $4(2.5)$ & $0(0.0)$ & $4(2.8)$ & 0.513 \\
\hline Ever & $33(20.6)$ & $7(46.7)$ & $26(18.1)$ & 0.009 \\
\hline Asthma, no. (\%) & $15(8.9)$ & $0(0.0)$ & $15(10.0)$ & 0.160 \\
\hline COPD, no. $(\%)$ & $9(5.3)$ & $3(16.7)$ & $6(4.0)$ & 0.024 \\
\hline \multicolumn{5}{|l|}{ Aetiology, no. (\%) } \\
\hline Idiopathic & $75(44.6)$ & $5(27.8)$ & $70(46.7)$ & 0.128 \\
\hline Post-TB & $42(25.0)$ & $6(33.3)$ & $32(21.3)$ & 0.250 \\
\hline Post-infectious & $38(22.6)$ & $5(27.8)$ & $37(24.7)$ & 0.773 \\
\hline FEV1 \% predicted (baseline), median (IQR) & $79(63-95)$ & $70(51-84)$ & $80(65-95)$ & 0.138 \\
\hline \multicolumn{5}{|l|}{ Spirometry pattern, no. (\%) } \\
\hline Normal & $52(39.0)$ & $3(25.0)$ & $49(40.5)$ & 0.294 \\
\hline Restrictive & $29(21.8)$ & $5(41.7)$ & $24(19.8)$ & 0.081 \\
\hline Obstructive & $15(11.2)$ & $1(8.3)$ & $14(11.6)$ & 0.735 \\
\hline Non-specific & $13(9.7)$ & $1(8.3)$ & $12(9.9)$ & 0.860 \\
\hline \multicolumn{5}{|l|}{ Microbiology, no. (\%) } \\
\hline Pseudomonas aeruginosa & $35(22.3)$ & $6(33.3)$ & $29(20.9)$ & 0.232 \\
\hline Pseudomonas aeruginosa colonisation & $24(15.2)$ & $5(29.4)$ & $19(14.4)$ & 0.113 \\
\hline Klebsiella pneumoniae & $16(10.2)$ & $1(5.6)$ & $15(10.8)$ & 0.490 \\
\hline Hemophilus influenzae & $6(3.8)$ & $1(5.6)$ & $5(3.6)$ & 0.683 \\
\hline Staphylococcus aureus & $10(6.4)$ & $3(16.7)$ & $7(5.0)$ & 0.057 \\
\hline NTM & $74(46.3)$ & $12(66.7)$ & $62(43.7)$ & 0.065 \\
\hline \multicolumn{5}{|l|}{ Radiology, no. (\%) } \\
\hline Upper lobes & $103(61.3)$ & $14(77.8)$ & $89(59.3)$ & 0.129 \\
\hline Middle lobes & $146(86.9)$ & $17(94.4)$ & $129(86.0)$ & 0.316 \\
\hline Lower lobes & $129(76.7)$ & $14(77.8)$ & $115(76.7)$ & 0.916 \\
\hline Lobes involved, median (IQR) & $3(2-4)$ & $4(3-4)$ & $3(2-4)$ & 0.004 \\
\hline Radiology severity score, median (IQR) & $6(4-9)$ & $7(4-9)$ & $6(5-6)$ & 0.007 \\
\hline Modified Reiff score, median (IQR) & $4(3-5)$ & $4(3-4)$ & $4(3-5)$ & 0.015 \\
\hline \multicolumn{5}{|l|}{ Radiology pattern, no. (\%) } \\
\hline Cylindrical & $132(78.5)$ & $12(66.7)$ & $120(80.0)$ & 0.193 \\
\hline Cystic & $22(13.0)$ & $5(27.8)$ & $17(11.3)$ & 0.051 \\
\hline Varicose & $14(8.3)$ & $1(5.6)$ & $13(8.7)$ & 0.652 \\
\hline Exacerbations in the past year, no. (\%) & $45(25.7)$ & $10(55.6)$ & $35(23.3)$ & 0.004 \\
\hline Haemoptysis ever, no. (\%) & $92(54.7)$ & $8(44.4)$ & $84(56.0)$ & 0.352 \\
\hline Significant haemoptysis, no. (\%) & $36(21.4)$ & $3(16.7)$ & $33(22.0)$ & 0.602 \\
\hline BSI score, median (IQR) & $6(5-8)$ & $8(6-9)$ & $6(5-8)$ & $<0.001$ \\
\hline CAT score, median (IQR) & $12.5(8.0-19.0)$ & $18.0(17.5-22.5)$ & $12.0(8.0-19.0)$ & 0.081 \\
\hline \multicolumn{5}{|l|}{ Aetiology of death in mortality group, no (\%) } \\
\hline Pneumonia & & & $4(22.2)$ & \\
\hline Bronchiectasis & & & $1(5.6)$ & \\
\hline Colorectal cancer & & & $1(5.6)$ & \\
\hline Coroner's case or unknown & & & $12(66.7)$ & \\
\hline
\end{tabular}

BMI: body mass index; BSI: Bronchiectasis Severity Index; CAT: COPD assessment test; COPD: chronic obstructive pulmonary disease; IQR: interquartile range; NCFB: non-cystic fibrosis bronchiectasis; NTM: non-tuberculous mycobacteria; TB: tuberculosis $P$ values in bold are significant 
accessible clinical score to aid in prognostication of patients with NCFB, which can influence clinical decision making and management. ${ }^{23}$ This was a composite score of clinical variables used to classify bronchiectasis severity, and prospectively validated to predict 1- and 4-year morbidity and mortality. The BSI scores, as well as chronic obstructive pulmonary disease (COPD) assessment test scores were obtained. ${ }^{23,25}$ Mortality outcome was defined in this study as the point of death from any cause. In calculating the clinical scores, missing data for variables were assumed to be normal. All data were entered into a secure digital platform (Research Electronic Data Capture).

Statistical analyses were done using SPSS Statistics software version 23.0 (IBM Corp, Armonk, US). Chi-square test and Mann-Whitney U test were applied in the comparison of categorical and continuous data, respectively. Data were expressed as median (interquartile range) for non-normally distributed continuous variables. Kaplan-Meier survival curves were plotted to determine the relationship between BSI severity grades and mortality, and hazard ratios were obtained using Cox proportional hazard regression models. Statistical significance was defined as a $P$ value less than or equal to 0.05 .

\section{RESULTS}

A total of 168 subjects were recruited. The clinical characteristics are presented in Table 1. There was preponderance of women $(67.8 \%)$ and Chinese ethnicity $(87.5 \%)$. The median age at diagnosis was 64 years (56-71). Most subjects were never-smokers $(76.9 \%)$. Nearly half the subjects had idiopathic bronchiectasis $(44.6 \%)$. Those with known aetiologies included 37 post-tuberculosis (TB) $(33.3 \%), 42$ post-infectious $(25.0 \%), 8$ autoimmune-related $(4.8 \%)$, 2 cilia dysmotility (1.2\%) and 2 immunoglobulin deficiency (1.2\%). One subject had alpha-1-antitrypsin level measured, which was normal. None underwent genetic testing for cystic fibrosis. The median follow-up period was 2.4 years $(1.3-3.4)$.

\section{Spirometry}

A normal spirometry was most commonly observed $(39.0 \%)$. Eleven percent of subjects had an obstructive pattern, and $21.8 \%$ showed restriction. The median forced expiratory volume in the first second (FEV1) predicted was $79 \%(63-95)$.

\section{Microbiology}

Thirty-five (22.3\%) subjects had at least 1 growth of Pseudomonas aeruginosa from a respiratory specimen.
Sixteen $(10.2 \%)$ subjects had Klebsiella pneumoniae and $6(3.8 \%)$ had Hemophilus influenzae. Seventyfour subjects $(46.3 \%)$ had sputum positive for NTM. The most common NTM isolated was Mycobacterium abscessus (41.9\%), followed by M. fortuitum (25.7\%), M. avium complex (20.3\%), and M. kansasii (8.1\%). Eighteen $(24.3 \%)$ subjects were initiated on NTM treatment, of whom 14 completed the course of treatment. Two patients did not complete treatment due to intolerable side effects, and 2 were still undergoing treatment at the time of this writing. The median treatment duration was 12 months (10-18).

\section{Radiology}

The median number of lobes involved was $3(2-4)$. The median modified Reiff score was 4 (3-5), and median radiology severity score was 6 (4-9). Majority had middle lobe involvement (86.9\%). The most common radiological pattern observed was a cylindrical pattern (78.5\%), followed by cystic pattern (13.0\%).

\section{Clinical history}

Fifty-seven subjects (33.9\%) had no history of exacerbations. Eight subjects $(4.8 \%)$ had 3 or more exacerbations per year in their lifetime. Seventy-two subjects $(60.0 \%)$ had a COPD assessment test score of 10 and above. Eighteen subjects $(10.7 \%)$ had demised during the period of follow-up. Ninety-two $(54.7 \%)$ subjects had a history of haemoptysis, of which 36 (21.4\%) were clinically significant.

\section{Sex differences}

There were more smokers $(51.8 \%$ vs $7.9 \%, P<0.001)$ and subjects with obstructive spirometry patterns $(20.8 \%$ vs $5.9 \%, P=0.009)$ observed among men (Table 2). Men were also more likely to have post-TB as the aetiology for bronchiectasis $(37.0 \%$ vs $15.8 \%$, $P=0.002)$ and upper lobe disease $(75.9 \%$ vs $54.4 \%$, $P=0.007$ ). Women had a higher prevalence of NTM (55.0\% vs $27.5 \%, P=0.001)$.

\section{Comparison of patients with and without a history of haemoptysis}

Ninety-two subjects (54.7\%) had a history of haemoptysis (Table 3). Subjects with haemoptysis tend to have a normal spirometry $(48.6 \%$ vs $27.9 \%$, $P=0.015)$ with higher baseline FEV1 (84L [67-100] vs $72 \mathrm{~L}$ [63-87], $P=0.004$ ), as compared to subjects without haemoptysis, who tend to have a restrictive pattern $(29.5 \%$ vs $15.3 \%, P=0.048)$. More subjects without haemoptysis had asthma $(14.5 \%$ vs $4.3 \%$, $P=0.022)$. The use of anticoagulation or antiplatelets was not associated with the development of haemoptysis. 
Table 2. Comparison of female and male NCFB patients in Singapore

\begin{tabular}{|c|c|c|c|}
\hline Characteristics & Female $(n=113)$ & Male $(n=54)$ & $P$ value \\
\hline Age, median (IQR), years & $62(56-69)$ & $69(60-74)$ & 0.115 \\
\hline BMI, median (IQR) & $19.3(17.4-21.2)$ & $19.1(17.0-26.2)$ & 0.658 \\
\hline \multicolumn{4}{|l|}{ Smoking status, no. (\%) } \\
\hline Active & $0(0)$ & $4(7.4)$ & 0.004 \\
\hline Previous & $9(7.9)$ & $24(44.4)$ & $<0.001$ \\
\hline Never & $97(85.8)$ & $24(44.4)$ & $<0.001$ \\
\hline \multicolumn{4}{|l|}{ Aetiology, no. (\%) } \\
\hline Idiopathic & $55(48.2)$ & $20(37.0)$ & 0.172 \\
\hline Post-infectious & $31(27.2)$ & $11(20.4)$ & 0.340 \\
\hline Post-TB & $18(15.8)$ & $20(37.0)$ & 0.002 \\
\hline \multicolumn{4}{|l|}{ Comorbidities, no. (\%) } \\
\hline Asthma & $11(9.6)$ & $4(7.4)$ & 0.634 \\
\hline COPD & $3(2.6)$ & $6(11.1)$ & 0.023 \\
\hline \multicolumn{4}{|l|}{ Spirometry, no. (\%) } \\
\hline Normal & $40(47.1)$ & $12(25.0)$ & 0.012 \\
\hline Restrictive & $19(22.4)$ & $10(20.8)$ & 0.838 \\
\hline Obstructive & $5(5.9)$ & $10(20.8)$ & 0.009 \\
\hline Non-specific & $7(8.2)$ & $6(12.5)$ & 0.426 \\
\hline FEV1 \% predicted (baseline), median (IQR) & $83(68-98)$ & $69(61-94)$ & 0.004 \\
\hline \multicolumn{4}{|l|}{ Sputum cultures, no. (\%) } \\
\hline Pseudomonas aeruginosa & $26(24.3)$ & $9(18.0)$ & 0.377 \\
\hline Klebsiella pneumoniae & $8(7.5)$ & $8(16.0)$ & 0.100 \\
\hline Hemophilus influenzae & $5(4.7)$ & $1(2.0)$ & 0.416 \\
\hline Staphylococcus aureus & $7(6.5)$ & $3(6.0)$ & 0.897 \\
\hline NTM & $60(55.0)$ & $14(27.5)$ & 0.001 \\
\hline \multicolumn{4}{|l|}{ Radiology, no. (\%) } \\
\hline Cylindrical & $90(78.9)$ & $42(77.8)$ & 0.863 \\
\hline Cystic & $14(12.3)$ & $8(14.8)$ & 0.649 \\
\hline Varicose & $10(8.8)$ & $4(7.4)$ & 0.765 \\
\hline \multicolumn{4}{|l|}{ Involvement, no. (\%) } \\
\hline Upper lobes & $62(54.4)$ & $41(75.9)$ & 0.007 \\
\hline Middle lobes & $102(89.5)$ & $44(81.5)$ & 0.152 \\
\hline Lower lobes & $88(77.2)$ & $41(75.9)$ & 0.856 \\
\hline Lobes involved, median (IQR) & $3(2-4)$ & $4(2-5)$ & 0.358 \\
\hline Radiology severity score, median (IQR) & $6(4-8)$ & $8(5-12)$ & 0.107 \\
\hline Modified Reiff score, median (IQR) & $4(2-5)$ & $4(3-6)$ & 0.263 \\
\hline Haemoptysis, no. (\%) & $64(56.1)$ & $28(51.9)$ & 0.602 \\
\hline Significant haemoptysis, no. (\%) & $29(25.4)$ & $7(13.0)$ & 0.066 \\
\hline BSI score, median (IQR) & $6(4-8)$ & $7(6-9)$ & 0.022 \\
\hline CAT score, median (IQR) & $13(8-20)$ & $13(7-18)$ & 0.394 \\
\hline
\end{tabular}

BMI: body mass index; BSI: Bronchiectasis Severity Index; CAT: COPD assessment test; COPD: chronic obstructive pulmonary disease; IQR: interquartile range; NCFB: non-cystic fibrosis bronchiectasis; NTM: non-tuberculous mycobacteria; TB: tuberculosis

$P$ values in bold are significant

\section{Mortality outcomes}

Causes of death are shown in Table 1. The most common aetiology of death was pneumonia $(4,22.2 \%)$. The cause of death was not available in 12 patients who died outside our institution. Our national policy on patient data confidentiality does not allow investigators to obtain information from national records for the purpose of research. Lower BMI, concomitant COPD, modified Reiff score, radiological severity, exacerbations and BSI scores correlated with mortality. Growth or colonisation of Pseudomonas aeruginosa was not associated with mortality. The hazard ratio for moderate grade BSI (BSI 5-8) compared to mild grade BSI (BSI 0-4) was 1.6 (confidence interval [CI] 0.188-15.053, $P=0.642$ ), and for severe grade $\mathrm{BSI}(\mathrm{BSI} \geq 9)$ compared to mild grade BSI was 14.8 (CI 1.929-114.235, $P=0.01$ ) (Table 4). The Kaplan-Meier survival curve 
Table 3. Comparison of NCFB patients with and without a history of haemoptysis

\begin{tabular}{|c|c|c|c|}
\hline Characteristics & Haemoptysis $(\mathrm{n}=92)$ & No haemoptysis $(n=76)$ & $P$ value \\
\hline Age, median (IQR), years & $65(56-71)$ & $63(56-73)$ & 0.554 \\
\hline BMI, median (IQR) & $19.0(16.9-21.1)$ & $20.2(17.9-23.4)$ & 0.247 \\
\hline Female gender, no. (\%) & $64(69.6)$ & $50(65.8)$ & 0.602 \\
\hline \multicolumn{4}{|l|}{ Smoking status, no. (\%) } \\
\hline Active & $3(3.4)$ & $1(1.4)$ & 0.438 \\
\hline Previous & $21(23.6)$ & $12(17.1)$ & 0.319 \\
\hline Never & $64(71.9)$ & $57(81.4)$ & 0.162 \\
\hline \multicolumn{4}{|l|}{ Aetiology, no. (\%) } \\
\hline Idiopathic & $41(44.6)$ & $34(44.7)$ & 0.982 \\
\hline Post-infectious & $21(22.8)$ & $21(27.6)$ & 0.474 \\
\hline Post-TB & $25(27.2)$ & $13(17.1)$ & 0.121 \\
\hline Asthma, no. (\%) & $4(4.3)$ & $11(14.5)$ & 0.022 \\
\hline COPD, no. (\%) & $6(6.5)$ & $3(3.9)$ & 0.461 \\
\hline \multicolumn{4}{|l|}{ Spirometry, no. (\%) } \\
\hline Normal & $35(48.6)$ & $17(27.9)$ & 0.015 \\
\hline Restrictive & $11(15.3)$ & $18(29.5)$ & 0.048 \\
\hline Obstructive & $7(9.7)$ & $8(13.1)$ & 0.538 \\
\hline Non-specific & $7(9.7)$ & $6(9.8)$ & 0.982 \\
\hline FEV1 \% predicted (baseline) & $84(67-100)$ & $72(63-87)$ & 0.004 \\
\hline \multicolumn{4}{|l|}{ Sputum cultures, no. (\%) } \\
\hline Pseudomonas aeruginosa & $22(24.7)$ & $13(19.1)$ & 0.403 \\
\hline Klebsiella pneumoniae & $8(9.0)$ & $8(11.8)$ & 0.569 \\
\hline Hemophilus influenzae & $3(3.4)$ & $3(5.9)$ & 0.736 \\
\hline Staphylococcus aureus & $8(9.0)$ & $2(2.9)$ & 0.124 \\
\hline NTM & $44(48.9)$ & $30(42.9)$ & 0.448 \\
\hline \multicolumn{4}{|l|}{ Radiology pattern, no. (\%) } \\
\hline Cylindrical & $73(79.3)$ & $59(77.6)$ & 0.787 \\
\hline Cystic & $8(8.7)$ & $14(18.4)$ & 0.063 \\
\hline Varicose & $11(12.0)$ & $3(3.9)$ & 0.062 \\
\hline \multicolumn{4}{|l|}{ Involvement, no. (\%) } \\
\hline Upper lobes & $53(51.5)$ & $50(48.5)$ & 0.305 \\
\hline Middle lobes & $81(55.5)$ & $65(44.5)$ & 0.488 \\
\hline Lower lobes & $71(55.0)$ & $58(45.0)$ & 0.544 \\
\hline Lobes involved, median (IQR) & $3(2-4)$ & $4(2-5)$ & 0.080 \\
\hline Radiology severity score, median (IQR) & $6(4-9)$ & $7(5-10)$ & 0.326 \\
\hline Modified Reiff score, median (IQR) & $4(2-5)$ & $4(3-6)$ & 0.073 \\
\hline Use of antiplatelets or anticoagulation, no. (\%) & $11(12.1)$ & $12(15.8)$ & 0.489 \\
\hline BSI score, median (IQR) & $7(5-9)$ & $6(4-8)$ & 0.398 \\
\hline CAT score, median (IQR) & $12(5-19)$ & $15(8-20)$ & 0.433 \\
\hline
\end{tabular}

BMI: body mass index; BSI: Bronchiectasis Severity Index; CAT: COPD assessment test; COPD: chronic obstructive pulmonary disease; IQR: interquartile range; NCFB: non-cystic fibrosis bronchiectasis; NTM: non-tuberculous mycobacteria; TB: tuberculosis

$P$ values in bold are significant

Table 4. Hazard ratios of Bronchiectasis Severity Index (BSI) severity grades

\begin{tabular}{lcc}
\hline BSI & Hazard ratios & 95\% confidence interval \\
\hline Grade 1: Mild & Reference & Reference \\
Grade 2: Moderate & 1.682 & $0.188-15.052$ \\
Grade 3: Severe & 14.844 & $1.929-114.235$ \\
\hline
\end{tabular}




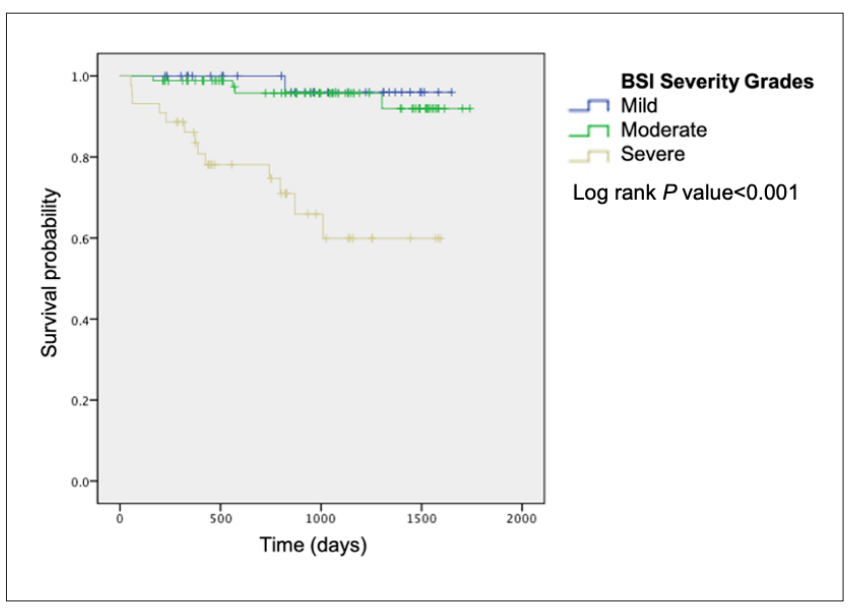

Fig. 1. Kaplan-Meier survival curves according to Bronchiectasis Severity Index (BSI) severity grades.

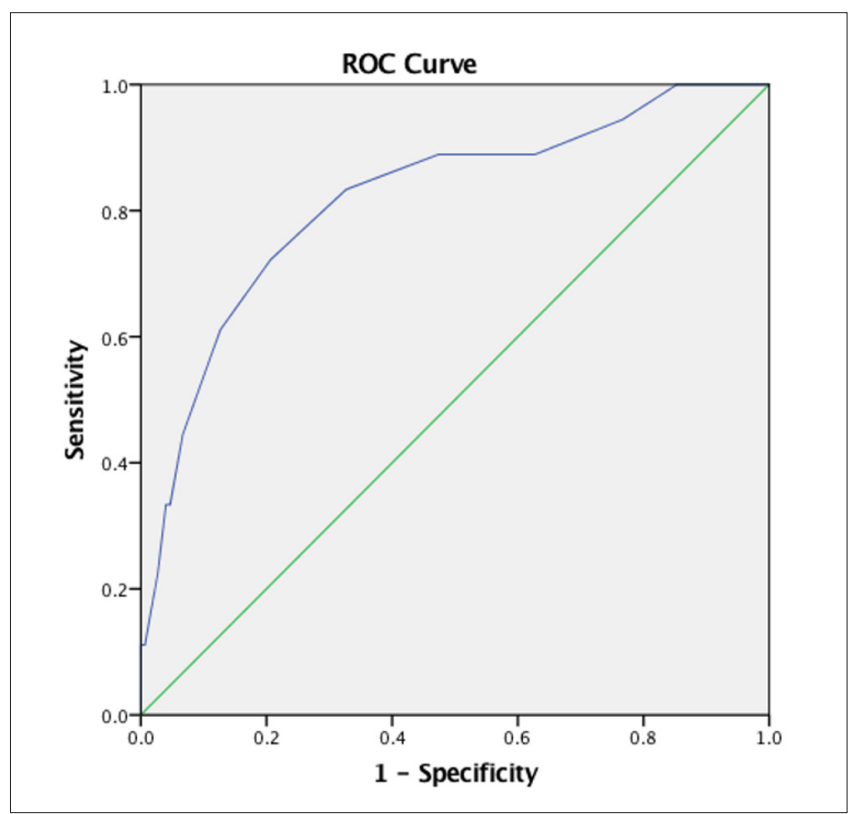

Fig. 2. Receiver operating characteristic curve for mortality according to Bronchiectasis Severity Index score.

ROC: receiver operating characteristic

demonstrated a lower survival at the median overall follow-up period of 2.4 years for subjects with severe BSI grade as compared to those with mild or moderate grades $(P<0.001)$ (Fig. 1). The receiver operating characteristic curve for mortality according to BSI score demonstrates an area under the curve of 0.818 (Fig. 2).

\section{DISCUSSION}

We observed a disproportionate Chinese majority and female predominance in our study, which is one of the first reports describing characteristics of NCFB patients in a multiethnic Southeast Asian population.
Most patients had normal spirometry patterns, and fewer than 2 exacerbations per year. There was a high prevalence of NTM, P. aeruginosa and K. pneumonia infection. Over half the patients had a history of haemoptysis, and approximately one-fifth of the patients had clinically significant haemoptysis. There is a higher proportion of NCFB among Chinese (87.5\%) compared to other races and this is out of proportion to Singapore's ethnic distribution $(74.3 \%$ Chinese, $13.5 \%$ Malays and $9.0 \%$ Indians, according to Singapore Department of Statistics' 2020 figures). The reason for this is unknown but postulated to be due to differences in disease aetiology and sputum microbiology. ${ }^{26} \mathrm{We}$ are unable to confirm these findings due to the small number of non-Chinese patients in our cohort.

Female preponderance of NCFB has been widely described in various global registries including the UK, US and Australia. ${ }^{10,13,18}$ Reasons postulated for this sex distribution include the smaller conducting airways in females, as well as the effects of oestrogen and progesterone on mucociliary clearance..$^{27}$ However, the sex ratio is reversed in TB-endemic countries like China, India and Pakistan. ${ }^{6}, 14,16$ In these countries, TB is a significant cause of NCFB and is more common among men. ${ }^{28} \mathrm{NCFB}$ patients in India and Pakistan are also younger- $83.1 \%$ of Pakistani patients are younger than 60, and the mean age of diagnosis in India was 56 (41-66) - compared to the European cohort with mean age of 67 (57-74)..$^{14,16}$ Factors such as the incidence of childhood pulmonary infections (including TB) and poor access to healthcare may contribute to the earlier onset, higher burden and increased severity of the disease. ${ }^{29,30}$ Southeast Asia accounts for over $40 \%$ of the global TB incidence. ${ }^{31}$ Despite a high incidence of TB in Singapore (47 per 100,000 population), the overall mean age of diagnosis of NCFB remains comparable to the UK and US. ${ }^{10,18}$ In the current study, $40 \%$ of the NCFB patients with known aetiology were due to previous TB infection. Idiopathic and post-TB bronchiectasis were equally common as aetiology of NCFB among male patients. On the other hand, the proportion of female patients with idiopathic bronchiectasis was thrice that of post-TB bronchiectasis. The higher frequency of male patients with post-TB bronchiectasis is likely due to a significantly higher TB prevalence among the men in Singapore $(70.7 \%$ vs $29.3 \%)^{32}$

Other significant sex differences observed were smoking status and sputum microbiology. Most female NCFB patients were never-smokers (90.7\%), compared to $46.2 \%$ in males $(P<0.001)$. This sex difference 
in smoking habits is similarly reflected in the larger proportion of COPD $(11.1 \%$ vs $2.6 \%, P=0.023)$, and obstructive spirometry $(20.8 \%$ vs $5.9 \%, P=0.009)$ seen in the male NCFB patients. The frequency of positive microbiology for NTM was significantly higher among females $(55.0 \%$ vs $27.5 \%, P=0.001)$.

The spirometry findings of the present study contradict previous reports that NCFB patients often have an obstructive lung pattern. ${ }^{33}$ Proposed mechanisms for the airway obstruction include collapse of the major airways during expiration, bronchial wall thickening, presence of endobronchial secretions and obliterative bronchitis. ${ }^{34}$ However, a high proportion of smokers in some older studies might have contributed to the high frequency of airway obstruction observed. Other studies assessing lung function in NCFB patients have reported that obstructive and restrictive spirometry were both associated with increased disease severity and hospitalisation rate. ${ }^{33}$ The high proportion of patients with normal spirometry and fairly preserved FEV1 may signal better clinical outcomes in our NCFB population.

Nearly half the NCFB patients $(46.3 \%)$ in our study had positive NTM culture, which is higher than the NTM prevalence (11.2\%) reported in China. ${ }^{6}$ Our NTM prevalence is more comparable to that of the US, which was $63 \%$ with a predominance of $M$. avium complex (37\%). ${ }^{10}$ In our population, M. abscessus was the most common mycobacterium species isolated $(18.6 \%)$. The most common bacterium isolated in our population is $P$. aeruginosa $(22.3 \%)$, which is comparable to other NCFB registries. ${ }^{10,14-16}$ Notably, $K$. pneumoniae is not widely reported in the microbiological characteristics of patients in US or European registries, and its overall incidence appears to be low. In our study, we observed an incidence of $10.2 \%$, comparable to the Thai (14\%) and Korean $(22.4 \%)$ cohorts. ${ }^{11,12} \mathrm{~K}$. pneumoniae is associated with less mortality, exacerbations and hospitalisation rates than $P$. aeruginosa. ${ }^{35-37}$ The lung microbiome composition appears to affect response to antiinflammatory therapy. In the erythromycin group in the Bronchiectasis and Low-dose Erythromycin Study (BLESS), patients with a Haemophilus-dominated microbiome had fewer exacerbations compared to those with Pseudomonas-dominated microbiome. ${ }^{38}$ There may be a geographical or racial predisposition that affects colonisation and further research into the bronchiectasis microbiome is imperative.

Interestingly, more than half of our patients $(54.7 \%)$ had a history of haemoptysis, $40 \%$ of whom were clinically significant. This is significantly higher than the prevalence of $20.9 \%$ and $23 \%$ that were reported in studies from Pakistan and the US, respectively. ${ }^{10,16}$ In bronchiectasis, chronic airway inflammation causes hypertrophy and tortuosity of the vessels accompanying the airways. ${ }^{39,40}$ Haemoptysis occurs due to the rupture of these vessels, usually in the setting of an acute infection or exacerbation. Despite our understanding of the pathophysiology, it remains uncertain why haemoptysis occurs more frequently in some patients but not others. We did not find an association between haemoptysis and the presence of hypertrophied bronchial arteries on computed tomography imaging in our population, which may suggest the presence of other factors affecting the development of haemoptysis. Other factors associated with haemoptysis in NCFB include the use of inhaled anticholinergics and short-acting beta agonists, ${ }^{41}$ presence of cystic pattern of on $\mathrm{CT}^{42}$ and post-TB aetiology ${ }^{37}$ However, these findings were not replicated in our study.

Lower BMI, smoking status, history of exacerbations, modified Reiff scores and radiological severity were associated with mortality in our study, which is in keeping with results from previous studies. ${ }^{23,43}$ BSI scores were calculated and showed to be a good predictor of mortality, with an area under the curve (AUC) value of 0.818 that was similar to the derivation and validation cohorts of the original study (AUC of 0.80 and $0.81-0.84$, respectively). The Kaplan-Meier survival curve and hazard ratios showed a significant difference in mortality when comparing patients with mild (BSI 0-4) and severe (BSI $>9$ ) bronchiectasis. Our results validate the use of BSI as a prognostic indicator in our local population. The inclusion of further markers of radiological severity such as a composite of lobar severity grades as we have done in our study may further refine the accuracy and utility of such clinical scores.

The limitations of this study include the relatively small cohort of patients, the potential selection bias due to recruitment of participants from a single study centre, and incomplete data, as in most real-world clinical studies. A multicentre study would likely provide more comprehensive information about characteristics of the NCFB population in Singapore. Causal inference between the variables analysed should also be made with caution given the relatively short follow-up period. The labels of post-infectious and post-TB aetiologies are based on self-reported histories of pulmonary infections or $\mathrm{TB}$, and may have an element of recall bias. However, as far as possible, 
objective evidence of previous pulmonary TB infection was documented. Similarly, there was a degree of reliance on self-reporting of bronchiectasis exacerbations and haemoptysis. We were unable to perform analysis for respiratory-specific mortality as the cause of death was not available for a significant proportion of the non-survivors. There may be interpretation bias introduced as the radiological assessment was performed by a sole radiologist. The strengths of the study include its prospective nature, and a broad inclusion criteria to reflect real-world clinical practice.

\section{CONCLUSIONS}

The NCFB population in Singapore has a female and Chinese predominance. Sex differences were found and haemoptysis was common. The BSI score is a useful predictor of mortality in our population. Future research and longitudinal data should focus on better understanding of the Asian bronchiectasis microbiome and cause of haemoptysis in NCFB.

\section{REFERENCES}

1. Chalmers JD, Chang AB, Chotirmall SH, et al. Bronchiectasis. Nat Rev Dis Prim 2018;4:45.

2. Quint JK, Millett ERC, Joshi M, et al. Changes in the incidence, prevalence and mortality of bronchiectasis in the UK from 2004 to 2013: A population-based cohort study. Eur Respir J 2016;47:186-93.

3. Henkle E, Chan B, Curtis JR, et al. Characteristics and Health-care Utilization History of Patients With Bronchiectasis in US Medicare Enrollees With Prescription Drug Plans, 2006 to 2014. Chest 2018; 154:1311-20.

4. Ringshausen FC, Rademacher J, Pink I, et al. Increasing bronchiectasis prevalence in Germany, 2009-2017: A populationbased cohort study. Eur Respir J 2019;54:2009-17.

5. Aliberti S, Sotgiu G, Lapi F, et al. Prevalence and incidence of bronchiectasis in Italy. BMC Pulm Med 2020;20:1-6.

6. Lin JL, Xu JF, Qu JM. Bronchiectasis in China. Ann Am Thorac Soc 2016;13:609-16

7. Choi H, Yang B, Nam H, et al. Population-based prevalence of bronchiectasis and associated comorbidities in South Korea. Eur Respir J 2019;54.

8. Yang B, Jang HJ, Chung SJ, et al. Factors associated with bronchiectasis in Korea: a national database study. Ann Transl Med 2020;8:1350.

9. Chandrasekaran R, Mac Aogáin M, Chalmers JD, et al. Geographic variation in the aetiology, epidemiology and microbiology of bronchiectasis. BMC Pulm Med 2018;18:1-14.

10. Aksamit TR, O'Donnell AE, Barker A, et al. Adult Patients With Bronchiectasis: A First Look at the US Bronchiectasis Research Registry. Chest 2017;151:982-92.

11. Palwatwichai A, Chaoprasong C, Vattanathum A, et al. Clinical, laboratory findings and microbiologic characterization of bronchiectasis in Thai patients. Respirology 2002;7:63-6.

12. Park J, Kim S, Lee YJ, et al. Factors associated with radiologic progression of non-cystic fibrosis bronchiectasis during long-term follow-up. Respirology 2016;21:1049-54.
13. Visser SK, Bye PTP, Fox GJ, et al. Australian adults with bronchiectasis: The first report from the Australian Bronchiectasis Registry. Respir Med 2019;155:97-103.

14. Dhar R, Singh S, Talwar D, et al. Bronchiectasis in India: results from the European Multicentre Bronchiectasis Audit and Research Collaboration (EMBARC) and Respiratory Research Network of India Registry. Lancet Glob Heal 2019;7:e1269-79.

15. Olveira C, Padilla A, Martínez-García M-Á, et al. Etiology of Bronchiectasis in a Cohort of 2047 Patients. An Analysis of the Spanish Historical Bronchiectasis Registry. Arch Bronconeumol (English Ed.) 2017;53:366-74.

16. Sharif N, Baig MS, Sharif S, et al. Etiology, Clinical, Radiological, and Microbiological Profile of Patients with Non-cystic Fibrosis Bronchiectasis at a Tertiary Care Hospital of Pakistan. Cureus 2020;12:e7208.

17. Lee H, Choi H, Sim YS, et al. KMBARC registry: protocol for a multicentre observational cohort study on non-cystic fibrosis bronchiectasis in Korea. BMJ Open 2020;10:1-8.

18. Chalmers JD, Aliberti S, Filonenko A, et al. Characterization of the "frequent exacerbator phenotype" in bronchiectasis. Am J Respir Crit Care Med 2018;197:1410-20.

19. Hill AT, Sullivan AL, Chalmers JD, et al. British thoracic society guideline for bronchiectasis in adults. Thorax 2019;74.

20. Polverino E, Goeminne PC, McDonnell MJ, et al. European Respiratory Society guidelines for the management of adult bronchiectasis. Eur Respir J 2017;50.

21. Miller MR, Hankinson J, Brusasco V, et al. Standardisation of spirometry. Eur Respir J 2005;26:319-38.

22. Reiff DB, Wells AU, Carr DH, et al. CT findings in bronchiectasis: Limited value in distinguishing between idiopathic and specific types. Am J Roentgenol 1995;165:261-7.

23. Chalmers JD, Goeminne P, Aliberti S, et al. The bronchiectasis severity index an international derivation and validation study. Am J Respir Crit Care Med 2014;189:576-85.

24. Hill AT, Haworth CS, Aliberti S, et al. Pulmonary exacerbation in adults with bronchiectasis: a consensus definition for clinical research. Eur Respir J 2017;49: 1700051.

25. Guan WJ, Chen RC, Zhong NS. The bronchiectasis severity index and FACED score for bronchiectasis. Eur Respir J 2016;47:382-84.

26. McShane PJ, Naureckas ET, Strek ME. Bronchiectasis in a diverse US population: Effects of ethnicity on etiology and sputum culture. Chest 2012;142:159-67.

27. Vidaillac C, Yong VFL, Jaggi TK, et al. Gender differences in bronchiectasis: A real issue? Breathe 2018;14:108-21.

28. Horton KC, MacPherson P, Houben RMGJ, et al. Sex Differences in Tuberculosis Burden and Notifications in Low- and Middle-Income Countries: A Systematic Review and Meta-analysis. PLoS Med 2016;13: e1002119.

29. Dhanaraj B, Papanna MK, Adinarayanan S, et al. Prevalence and risk factors for adult pulmonary tuberculosis in a metropolitan city of South India. PLoS One 2015;10: e0124260.

30. Central TB Division. Revised National Tuberculosis Control Programme Annual Status Report. India TB Report 2018, March 2018. Available at: https://tbcindia.gov.in/showfile.php?lid=3314. Accessed on 28 May 2021

31. World Health Organization. WHO: Tuberculosis Country Profiles 2018. Available at: https://www.who.int/tb/publications/global_report/ tb19 Report country profiles 15 October2019.pdf?ua=1. Accessed on 28 May 2021.

32. Jappar SB, Low SY. Tuberculosis trends over a five-year period at a tertiary care university-affiliated hospital in Singapore. Singapore Med J 2015;56:502-5. 
33. Stretton R, Poppelwell L, Salih W, et al. Patterns of spirometry in bronchiectasis patients and relationship to markers of disease severity and hospitalisation. Eur Respir J 2013;42:P2695.

34. Chalmers JD, Aliberti S, Blasi F. Management of bronchiectasis in adults. Eur Respir J 2015;45:1446-62.

35. Finch S, McDonnell MJ, Abo-Leyah H, et al. A Comprehensive Analysis of the Impact of Pseudomonas aeruginosa Colonization on Prognosis in Adult Bronchiectasis. Ann Am Thorac Soc 2015; 12:1602-11.

36. King PT, Holdsworth SR, Freezer NJ, et al. Microbiologic follow-up study in adult bronchiectasis. Respir Med 2007;101:1633-8.

37. Davies G, Wells AU, Doffman S, et al. The effect of Pseudomonas aeruginosa on pulmonary function in patients with bronchiectasis. Eur Respir J 2006;28:974-9.

38. Serisier DJ, Martin ML, Mcguckin MA, et al. Effect of Long-term, Low-Dose Erythromycin on Pulmonary Exacerbations Among
Patients With Non-Cystic Fibrosis Bronchiectasis. JAMA 2013; 309:1260-7.

39. Martin LN, Higgins L, Mohabir P, et al. Bronchial Artery Embolization for Hemoptysis in Cystic Fibrosis Patients: A 17-Year Review. J Vasc Interv Radiol 2020;31:331-5.

40. Dave BR, Sharma A, Kalva SP, et al. Nine-year single-center experience with transcatheter arterial embolization for hemoptysis: medium-term outcomes. Vasc Endovascular Surg 2011;45:258-68.

41. Jang EJ, Lee C-H, Yoon H Il, et al. Association between inhaler use and risk of haemoptysis in patients with non-cystic fibrosis bronchiectasis. Respirology 2015;20:1213-21.

42. Guan WJ, Yuan JJ, Gao YH, et al. Hemoptysis in adults with bronchiectasis: correlation with disease severity and exacerbation risk. Zhonghua Jie He He Hu Xi Za Zhi 2017;40:16-23.

43. Ellis HC, Cowman S, Fernandes M, et al. Predicting mortality in bronchiectasis using bronchiectasis severity index and FACED scores: A 19-year cohort study. Eur Respir J 2016;47:482-9. 